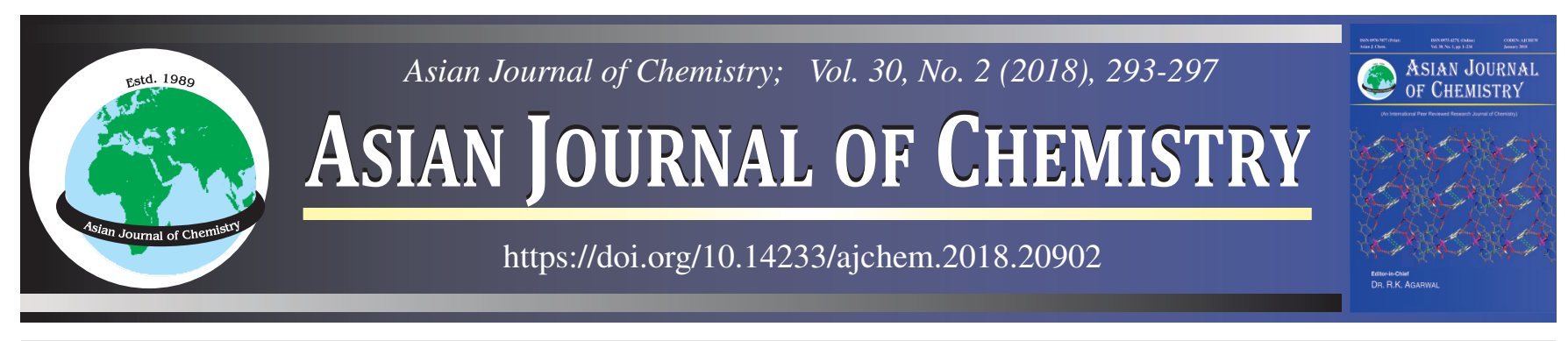

\title{
Development of Solid Lipid Nanoparticles of Gac (Momordica cocochinensis Spreng) Oil by Nano-Emulsion Technique
}

\author{
H.C. MAI ${ }^{1, *}$, T.T.T. LE ${ }^{1}$, T.T. DieP ${ }^{1}$, T.H.N. LE ${ }^{2}$, D.T. NGUYEN ${ }^{3}$ and L.G. BACH ${ }^{3}$
}

${ }^{1}$ Department of Chemical Engineering and Processing, Nong Lam University, Ho Chi Minh City, Vietnam

${ }^{2}$ Ho Chi Minh City University of Technology, VNU-HCM, Ho Chi Minh City, Vietnam

${ }^{3}$ NTT of Institute of Hi-Technology, Nguyen Tat Thanh University, Ho Chi Minh City, Vietnam

*Corresponding author: Tel/Fax: +84 28 37245030; E-mail: maihuynhcang@hcmuaf.edu.vn

Solid lipid nanoparticles have emerged as a next-generation drug delivery system with potential applications in the pharmaceutical field, cosmetics, research, clinical medicine and other allied sciences. In the present work, gac oil-loaded solid lipid nanoparticles were successfully prepared by a nano emulsion technique employing high-speed homogenizer. In this study, solid lipid nanoparticle were successfully prepared by using Emulgade SE-PF ${ }^{\mathrm{TM}}$ lipid, high-speed homogenization with the support of surfactant. The suspensions contained $5 \%$ active agents (gac oil w/w) were dispersed in the presence of surfactant (Tween-80:Span-80 ratio of 72:28 w/w) at the content of $5 \%$ by using the IKA homogenizer at the speed of $10000 \mathrm{rpm}$ for $60 \mathrm{~min}$ ( $30 \mathrm{~min}$ hot homogenization and $30 \mathrm{~min}$ cold homogenization). The obtained mixture had the median size of $200 \mathrm{~nm}$ (by laser diffractometric spectrometry, LSD). Entrapment efficiency was about $90.8 \%$.

Keywords: Solid lipid nanoparticles, Gac oil (Momordica cochinchinensis Spreng.), Homogenization, Emulgade SE-PF ${ }^{\mathrm{TM}}$.

\section{INTRODUCTION}

Gac fruit is rich in carotenoid compounds, especially $\beta$ carotene and lycopene, which are highly antioxidant activity and ability of anti-aging, skin tanning, skin care and skin protection. In gac aril, the content of $\beta$-carotene, a pro-vitamin A compound, is 1.8 times higher than that in cod-liver oil, 15 times higher than that in carrots. The pure natural $\beta$-carotene has the strongest anti-aging effect. Together with this, vitamin A supplementation helps to maintain a healthy skin as well as to enhance skin elasticity and moisture. In gac, lycopene content is 70 times higher than that in tomatoes, so that lycopene particles can crystallize themselves [1-4]. This type of carotenoid has a strong anti-aging and is the only carotenoid that can prevent heart attacks and protect gene from damage. From these advantages, recent researchers have found to incorporate carotenoids into cosmetics such as skin lotions in order to enhance the ability of skin protection. However, the drawback of carotenoids is extremely sensitive to temperature, light and oxygen; thus, this compound is easily decomposed $[5,6]$.

Colloidal drug delivery systems, namely, oil-in-water emulsions, liposomes, micelles, microparticles and nano- particles, offer new opportunities for targeting drugs, pharmaceuticals and cosmetics. Introduced in 1991, solid lipid nanoparticles (SLNs) have demonstrated superiority in certain features over other types of colloidal carriers. Solid lipid nanoparticles comprise spherical lipid particles in the nanometer size range of 50-1000 $\mathrm{nm}$ [7-9]. They offer various advantages in drug delivery due to their small particle size, large surface area as well as ability to modify their surface properties easily. Solid lipid nanoparticle systems can also solubilize poorly water-soluble compounds and provide controlled release [10]. Moreover, solid lipid nanoparticles provide both stability of solid matrix and biological compatibility of lipid carriers while avoiding the shortcomings of liposomes, which include undesired stability problems and polymeric particles, which are subject to the potential toxicity of the materials [11].

Developing solid lipid nanoparticles containing gac oil is a new approach to enhance the activity of the carotenoids as well as increase their durability when blended into cosmetic products.

Herein, the aim of this study was to investigate the ability of solid lipid nanoparticles in gac oil encapsulation. The effects of homogenization conditions on solid lipid nanoparticles containing gac oil production were investigated. 


\section{EXPERIMENTAL}

Gac oil was obtained from Gac Viet Company, Vietnam. Emulgade SE-PF ${ }^{\mathrm{TM}}$ (glyceryl stearate, ceteareth-20, ceteareth12 , cetearyl alcohol, cetyl palmitate) was purchased from Cognis Deutschland GmbH \& Co. KG Care Chemicals. All other chemicals including Tween- 80 and Span- 80 were of analytical grade.

Preparation of gac oil-loaded solid lipid nanoparticles: According to the procedure developed by our group, solid lipid nanoparticles containing gac oil were prepared by high-pressure homogenization including two steps: hot and cold homogenization $[12,13]$. The ratio of gac oil:solid lipid (Emulgade): Tween-80:Span-80:water was 5:2.5:3.6:1.4:87.5. In the first stage, the solid lipid was melted to form a lipid phase and kept at proper temperature. Meanwhile, deionized water containing two surfactants (Tween-80 and Span-80) was also heated to the same temperature as the molten lipid phase. The molten lipid phase was mixed with the heated aqueous solution and then stirred by stirring instrument for $15 \mathrm{~min}$. Then, the mixture underwent high-shear dispersion with a high-speed homogenizer (IKA, Germany) at speed and time investigated to form a pre-emulsion.

For cold homogenization, the temperature of resulting preemulsion was cool down to $0-5^{\circ} \mathrm{C}$ by placing into an ice bath. Then, the mixture continually was homogenized with speed and time, which are similar to hot homogenization. The solid lipid nanoparticles were then characterized in terms of their particle size distribution, particle morphology, entrapment efficiency as well as stability during different storage conditions.

Particle size determination: The mean particle size and size distribution of gac oil-loaded solid lipid nanoparticles were measured using a dynamic light scattering instrument (DLS) (Horiba LA920, Japan). All analyses were performed in automeasuring mode at $25^{\circ} \mathrm{C}$ and the results were presented as the average value of triplicate samplings and measurement for each formulation.

Gac oil entrapment efficiency: The gac oil entrapment efficiency was identified as reported previously. Briefly, a $5 \mathrm{~mL}$ aliquot of gac oil-loaded solid lipid nanoparticles was added to $5 \mathrm{~mL}$ of $n$-hexane and the mixture was vortex mixed. Then, the mixture was centrifuged at $5000 \mathrm{rpm}$ for $10 \mathrm{~min}$ to separate the undissolved components and the supernatant containing the gac oil extracted from solid lipid nanoparticles was analyzed using an HPLC method. The blank sample was also prepared by the method mentioned above without gac oil. The entrapment efficiency (EE \%) of solid lipid nanoparticles was calculated by the equation as follow:

$$
\mathrm{EE}(\%)=\frac{\left(\mathrm{DG}_{\text {blank }}-\mathrm{DG}_{\mathrm{gac}}\right)}{\mathrm{DG}_{\text {blank }}} \times 100
$$

where $\mathrm{DG}_{\text {blank }}$ and $\mathrm{DG}_{\mathrm{gac}}$ represent a number of active ingredients extracted from blank and gac oil-loaded solid lipid nanoparticles samples, respectively.

\section{RESULTS AND DISCUSSION}

Effects of hot homogenization speed: Fig. 1 presents influences of various hot homogenization speed on size distribution of solid lipid nanoparticles containing gac oil.

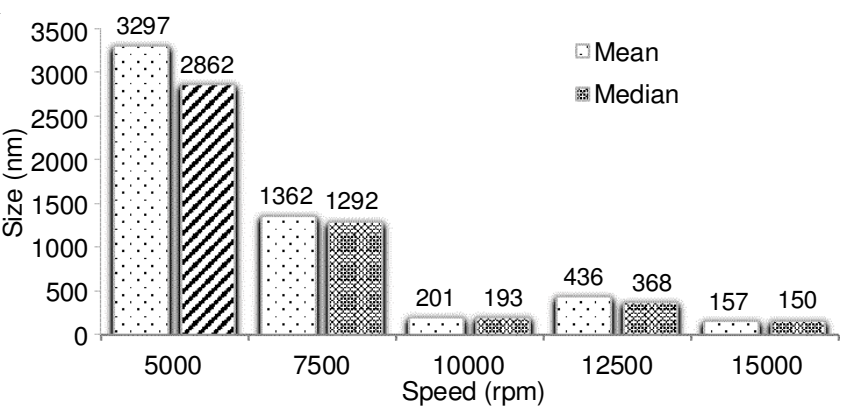

Fig. 1. Effects of hot homogenization speed on size distribution of gac oil-loaded solid lipid nanoparticles

Based on the results (dynamic light scattering measurement), the hot homogenization speed significantly influenced the size and distribution of solid lipid nanoparticles. When the speed of hot homogenization increased from 5000 to $7500 \mathrm{rpm}$, the particle size decreased by more than half (from 3297 to 1362 $\mathrm{nm})$. When the speed increased to $10000 \mathrm{rpm}$, the particle size decreased significantly and the solid lipid nanoparticles system had an impressive size: $201 \mathrm{~nm}$ in average and uniform distribution. This was an ideal dispersion system for skin care products as this size was suitable for active ingredient-loaded solid lipid nanoparticles easily penetrating deep into the skin, nourishing as well as protecting skin. Meanwhile, the smaller size could cause cytotoxicity since this was not possible to control the absorption content. Moreover, the higher homogenization speed, the more uniform of size distribution (similar mean and median values).

This result is perfectly consistent with the trend as the more energy is supplied, the smaller average size. The energy provided to the system not only broke down the big particles into small ones but also prevented adhesion between the oil particles. Therefore, when the speed of hot homogenization increases to an appropriate value, the dispersion ability of oil droplets is much easier, resulting in smaller particle sizes. However, if the speed increases further, the oil particles are more likely to collide and aggregate with others, leading to an increase in particle size. More specifically, the homogenization speed increased further up to $12500 \mathrm{rpm}$, the particle size increased between 200 and $436 \mathrm{~nm}$. The suspension system was kept at 5-10 ${ }^{\circ} \mathrm{C}$ avoiding light conditions and continue to be evaluated for physical and chemical durability.

The physical durability of gac oil-loaded was assessed through the size distribution and average size during the storage periods of 5, 15 and 30 days that was shown in Fig. 2. Based on the results, the higher homogenization speed (from 5000 to $10000 \mathrm{rpm}$ ), the more stable the dispersion system. At the speed of $5000 \mathrm{rpm}$, the particle size considerably varied with a downward trend. This might be due to the larger particle size, the stronger effect of low-temperature storage. On the other hand, the reduction of average size might be due to the fact that when the particles were agglomerated, the energy was insufficient to separate individual particle. However, the solid lipid system gradually stabilized over time, the particles separated in a stable state. The gac oil-load solid lipid nanoparticles had the highest stability after homogenized at 10000 rpm, the particle size was almost unchanged during the storage period. When the homogenization speed increased to 12500 


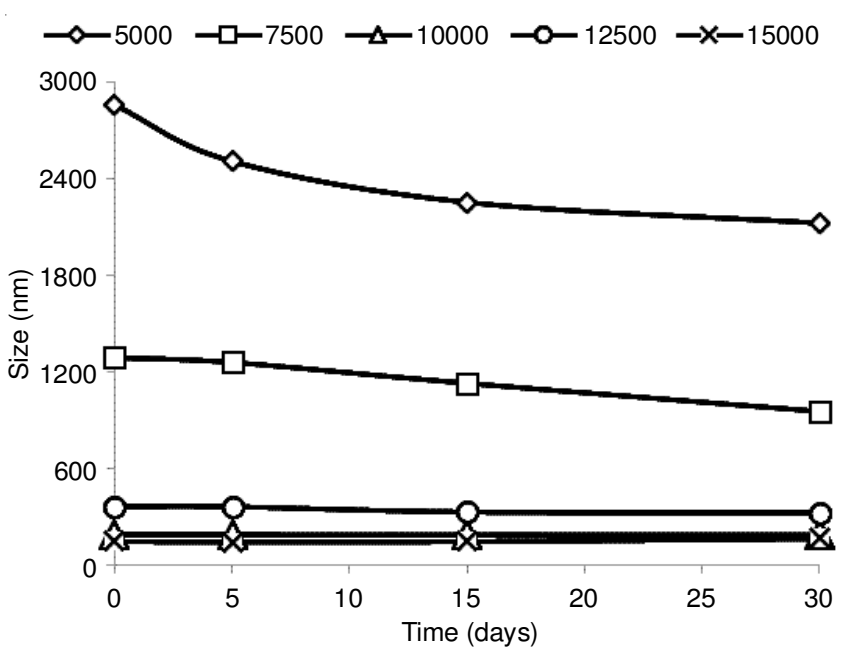

Fig. 2. Size change according to homogenization speeds

and $15000 \mathrm{rpm}$, solid lipid nanoparticle containing gac oil remained stable after 30 days of storage.

The chemical durability was evaluated through colour measurement method by the LCh system. Suspension samples produced in the above experiment were wrapped to avoid light and stored at $10{ }^{\circ} \mathrm{C}$. Then, colour variations of these samples were identified through $\Delta \mathrm{E}$ during the storage period. From this, the efficiency of encapsulation of solid lipid nanoparticles was predicted. When the carotenoid changed, the colour tends to fade resulting in a decrease of suspension colour; that caused an increase of colour variation. The results show that colour variation increased during storage period and colour variation was also greatly affected by the homogenization condition.

According to Fig. 3, the change in the $\Delta \mathrm{E}$ value during storage period showed that the higher homogenization speed, the better colour stability. At the speed of $5000 \mathrm{rpm}$, the value of $\Delta \mathrm{E}$ sharply and significantly increased and after 30 days of storage, this value reached 70 . This result presents that the encapsulation ability of solid lipid nanoparticles was inefficient, most of gac oil particles were in free form. Hence, these particles were going to be decomposed during preservation time. When the homogenization speed was increased to 7500 $\mathrm{rpm}$, the value of $\Delta \mathrm{E}$ changed significantly compared to 5000 $\mathrm{rpm}$. And if the speed continued to increase to $10000 \mathrm{rpm}$, the system was almost stable in colour. After 28 days, the increase of $\Delta \mathrm{E}$ value was only under 5 ; that meant under this condition, the solid lipid nanoparticles considerably entrapped the active substance and the gac oil was protected in the solid lipid shell so it was not affected by storage period. However, if the speed continued to increase to $12500 \mathrm{rpm}$, the $\Delta \mathrm{E}$ value increased significantly. This was probably due to the high homogenization speed leading to the broken down solid lipids, whereby the gac oil was pushed out of the solid lipid and partly in the free form. Hence, with hot homogenization speed of 10000 $\mathrm{rpm}$, the gac oil-loaded solid lipid nanoparticles achieved small size, durability as well as efficient encapsulation (according to LCh colour method).

Effects of hot homogenization time: Fig. 4 shows that the longer time of hot homogenization, the lower particle size. This was because the shorter times (10 and $20 \mathrm{~min}$ ) were not enough to disperse the oil particles down to the nanoscale, therefore

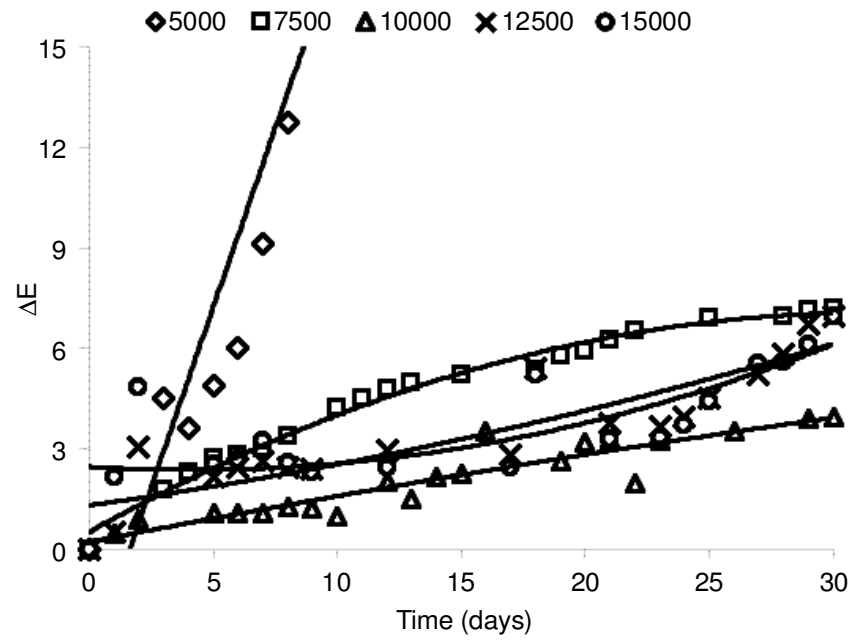

Fig. 3. $\Delta \mathrm{E}$ change at different storage periods at different homogenization speeds

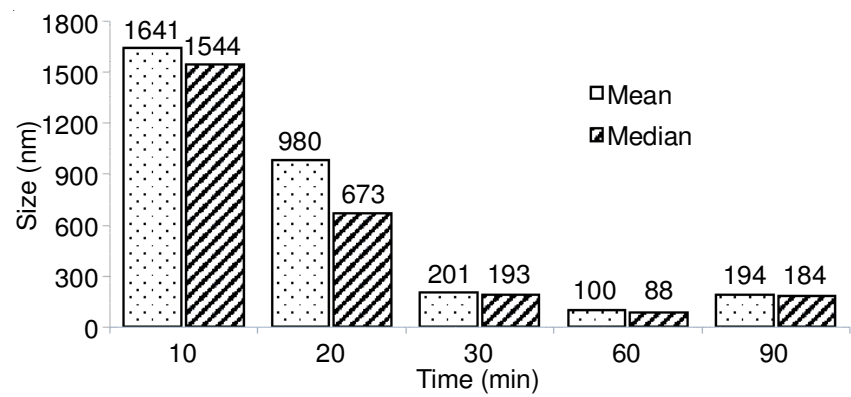

Fig. 4. Size distribution of gac oil-loaded solid lipid nanoparticles at different hot homogenization times

the system owned relatively large size (1641 and $980 \mathrm{~nm})$. By increasing the time to $30 \mathrm{~min}$, the particle size decreased significantly to $201 \mathrm{~nm}$ and the system was in more uniform distribution (same median and mean values). When the homogenization times reached $60 \mathrm{~min}$, the particle size reduced a half to $100 \mathrm{~nm}$. However, when the time increased to $90 \mathrm{~min}$, the particle size increased. This was due to prolonging the homogenization time at $80^{\circ} \mathrm{C}$, the oil particles tended to collapse and aggregate together. The sample was kept at a temperature of 5-10 ${ }^{\circ} \mathrm{C}$ avoiding light conditions and continue to be evaluated for physical and chemical durability.

It is clear that the longer time of hot homogenization time, the more stable particle size (Fig. 5). Similar to the experiment of homogenization speed change, samples with medium size particles tended to collapse and reduce the size when stored at low temperature over time. Two samples homogenized at 10 and 20 min suddenly decreased particle size after 5 storage days, but after that the particle size of this sample was stable. This might due to the fact that when the particles were agglomerated, the energy was not enough to separate individual particle. However, prolonging storage time, the dispersion system started to stabilize, the particles separated individually. Meanwhile, samples homogenized at 30,60 and $90 \mathrm{~min}$, were stable until the 30th day. Among these samples, nanoparticles homogenized at 30 and $90 \mathrm{~min}$ had the highest stability, particle size almost unchanged after 30 days of storage.

From the $\Delta \mathrm{E}$ values in Fig. 6, it can be seen that the longer storage time, the greater colour variation. This was due to the 


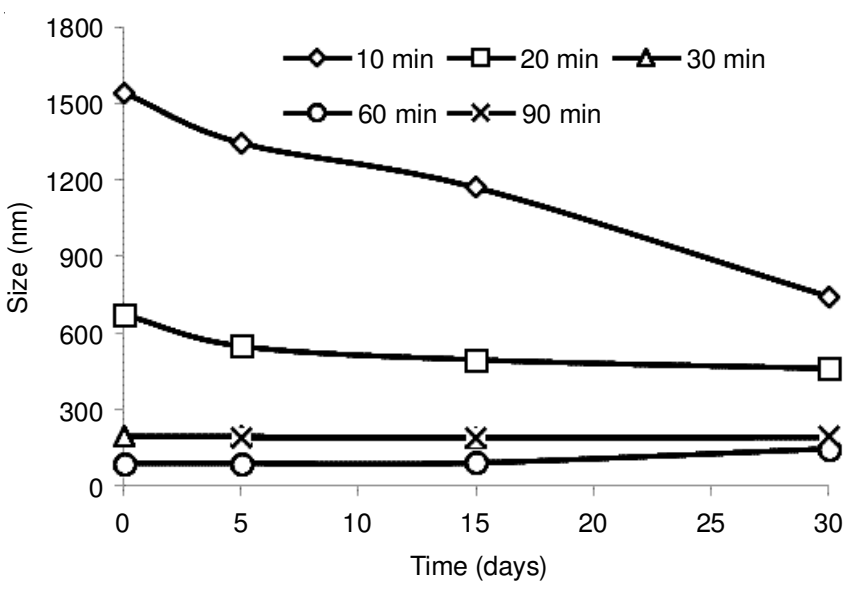

Fig. 5. Size change according to storage periods at different hot homogenization times

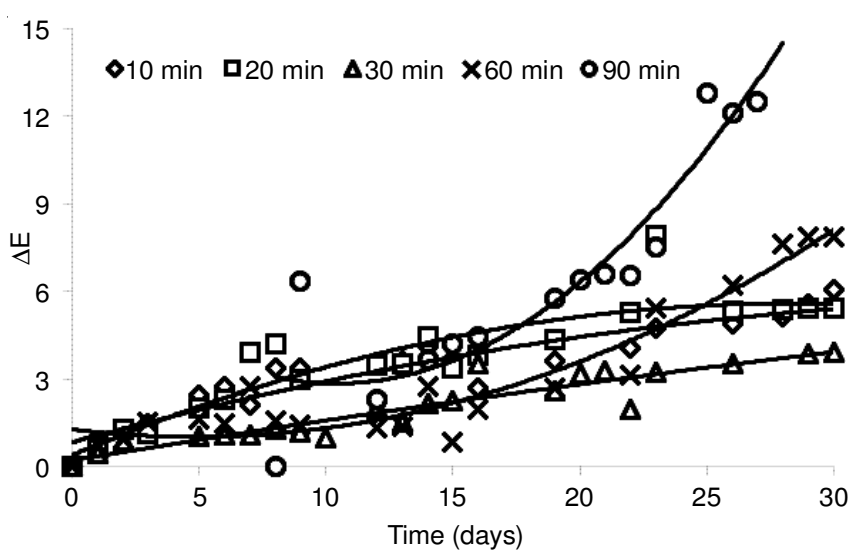

Fig. 6. $\Delta \mathrm{E}$ change at different hot homogenization times

modification of carotenoids leading to the light colour of the suspension. The $\Delta \mathrm{E}$ values of samples homogenized at 10 , 20, 60 and $90 \mathrm{~min}$ had a fluctuating trend. Especially, at 90 min of homogenization, the $\Delta \mathrm{E}$ value changed significantly. The reason might be that longer homogenization time caused the extremely small particle size. This led to a decrease of inside volume of the solid lipid nanoparticles containing gac oil that could not encapsulate totally gac oil. Hence, the ability of entrapment and protection of solid lipid nanoparticles was reduced remarkably. At the hot homogenization time of 30 min, the minimum change in $\Delta \mathrm{E}$ value gained 3.94 after 30 days of storage. This indicated a high encapsulation ability and a more stable chemical stability. Therefore, a short or long hot homogenization affect the protective effect of solid lipid nanoparticles and the short homogenization time was not sufficient for encapsulation of active ingredient while the long homogenization caused small particles resulting in a decrease of the volume containing active ingredients.

Effects of cold homogenization time: Cold homogenization is an important process in making solid lipid nanoparticles because when the hot homogenization is completed, the system temperature is reduced to $0-5^{\circ} \mathrm{C}$, the lipid oil begins to solidify. Thus, the time required for cold homogenization should be appropriate so that the solid lipids are separated to a smaller size. This directly and significantly affects the solid lipid nanoparticles size. The results in Fig. 7 show that when the homogenization time increased from 10 to $20 \mathrm{~min}$, the mean value of

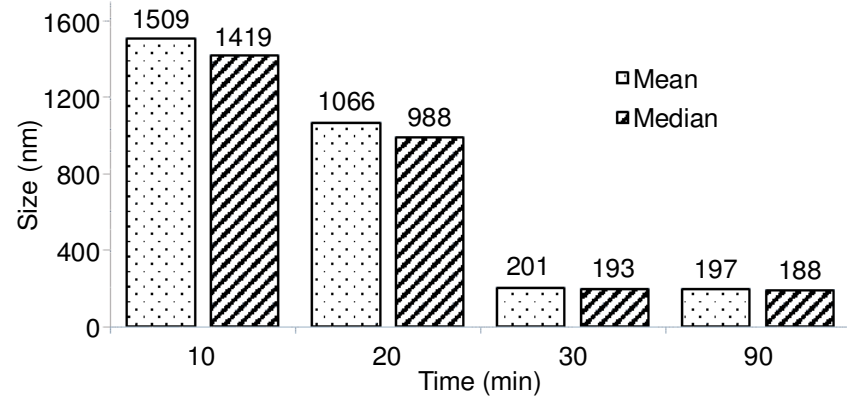

Fig. 7. Size distribution of gac oil-loaded solid lipid nanoparticles at different cold homogenization times

solid lipid nanoparticles decreased between $1509 \mathrm{~nm}$ and 1066 $\mathrm{nm}$. However, the particle size was still large, hence $20 \mathrm{~min}$ time was not sufficient to homogenize the solid lipid particles. By contrast, increasing the time to $30 \mathrm{~min}$, the particle size dramatically reduced to an impressive mean value of $201 \mathrm{~nm}$. This indicated that the cold homogenization time significantly influenced the particle size. The particle size decreased 5 times when only increasing the time of $10 \mathrm{~min}$. However, when the time was prolonged to $90 \mathrm{~min}$, the particle size decreased in considerably to $196 \mathrm{~nm}$. Because of its orientation in cosmetic applications, the particle size of about $200 \mathrm{~nm}$ was appropriate and did not need to be further reduced. Also, increasing the time required more energy consumption. Therefore, the cold homogenization time of $30 \mathrm{~min}$ was suitable.

Fig. 8 shows that the cold homogenized samples for 10 and $20 \mathrm{~min}$ had low stability. The reason was that the large particles stored at low temperatures for long periods caused contraction phenomenon, resulting in reduced size and unstable system. As time increased, particle size distribution was more stable. At cold homogenization time of 30 and $90 \mathrm{~min}$, the samples had better stability and colour difference was insignificant after storage of 30 days. Therefore, the cold homogenization of $30 \mathrm{~min}$ was the most appropriate process for time-consuming, the energy, proper size and uniform dispersion.

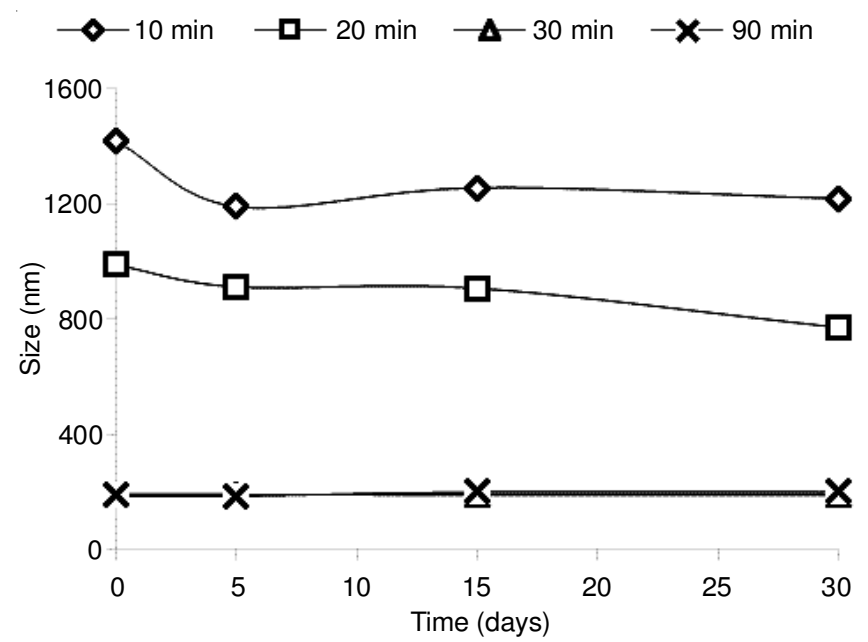

Fig. 8. Size change according to storage periods at different cold homogenization times

Similar to hot homogenization time, $\Delta \mathrm{E}$ value of cold homogenization samples also had the same trend (Fig. 9). The longer cold homogenization time, the more stable colour system over 


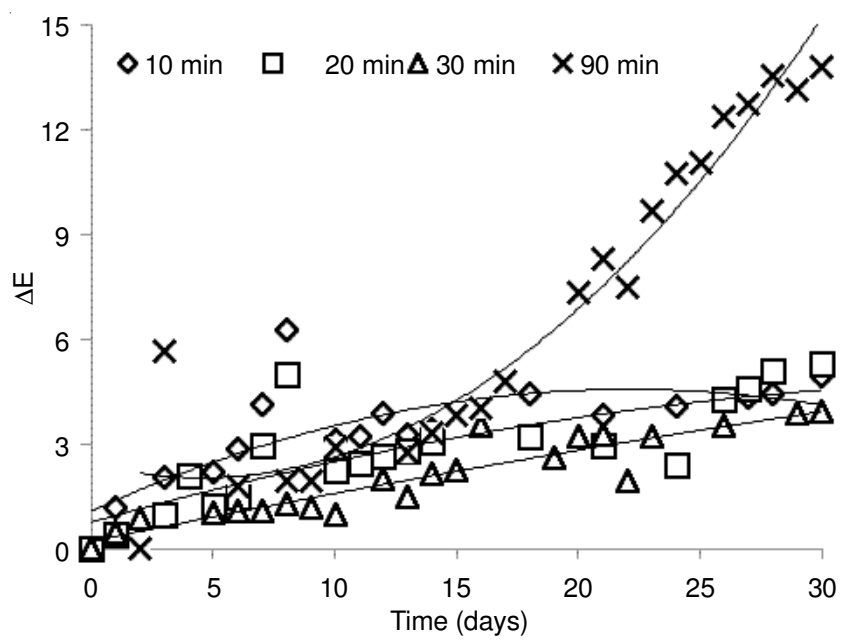

Fig. 9. $\Delta \mathrm{E}$ change at different cold homogenization times

time. However, the best homogenization time was $30 \mathrm{~min}$ as the time increased to $90 \mathrm{~min}$, the particle size decreased, the volume contained active ingredients was too small. Hence, the encapsulation efficiency of active ingredients decreased, leading to an unusually rapid increase of $\Delta \mathrm{E}$ value after 30 days of storage.

Gac oil entrapment efficiency: The HPLC method was used to quantitate lycopene content at two different samples and from this, the encapsulation efficiency was calculated. Encapsulation efficiency was observed to be $90.82 \%$ (Table$1)$. This indicated that the lycopene was greatly encapsulated, the free gac oil content was less than $10 \%$. Hou et al. [12] prepared solid lipid nanoparticles containing mifepristone with the following materials: glycerol monostearate, Tween-80 and glycerol [12]. The LDS results of this research were $106 \mathrm{~nm}$ for particle size and 87, $89 \%$ for entrapment efficiency. In 2015, Le and Phan [14] had successfully produced solid lipid nanoparticle system containing orange oil with the average size of $80 \mathrm{~nm}$. To determine the amount of essential oil encapsulated within the solid lipid nanostructure, the suspension was coldly filtered and then washed 3 times with distilled water, in order to obtain solid lipid particles. Then, the system was distilled by steam and obtained a volume of essential oil inside solid lipid particles. The encapsulation efficiency of solid lipid nanoparticles containing orange oil was $40 \%$ while this parameter for gac oil was $90.82 \%$.

TABLE-1
GAC OIL ENTRAPMENT EFFICIENCY OF
SOLID LIPID NANOPARTICLES

\section{Conclusion}

The optimum conditions for the formation of solid lipid nanoparticles were the homogenization speed of $10000 \mathrm{rpm}$, the hot homogenization time of $30 \mathrm{~min}$ and, the cold homogenization time of $30 \mathrm{~min}$. The surfactant content was $5 \%$ (Tween-80:Span-80 of 72:28 w/w), emulgade content was $2.5 \%$ and gac oil content was $5 \%$. The gac oil-loaded solid lipid nanoparticles owned the average size of $200 \mathrm{~nm}$. The carotenoid encapsulation efficiency of solid lipid nanoparticles was considerably high with over $90 \%$.

\section{ACKNOWLEDGEMENTS}

The researches were funded by Ministry of Education and Training, Vietnam.

\section{REFERENCES}

1. G. Schnurr, N. Misawa and G. Sandmann, Biochem. J., 315, 869 (1996); https://doi.org/10.1042/bj3150869.

2. J. Shi, Crit. Rev. Biotechnol., 20, 293 (2000); https://doi.org/10.1080/07388550091144212.

3. N.I. Krinsky, Exp. Biol. Med., 218, 95 (1998) https://doi.org/10.3181/00379727-218-44273.

4. L. Cao-Hoang, H. Phan-Thi, F.J. Osorio-Puentes and Y. Waché, Food Res. Int., 44, 2252 (2011);

https://doi.org/10.1016/j.foodres.2011.03.036.

5. E.S. Curtis, R. Kalafsky and E.R. Kaplan, Method of Treating the Skin with Organic Acids in Anhydrous Microsphere Delivery Systems, US Patent 5962018 A (1999).

6. M.E. Darvin, W. Sterry, J. Lademann and T. Vergou, Molecules, 16, 10491 (2011); https://doi.org/10.3390/molecules161210491.

7. M.D. Triplett, Ph.D. Thesis, Department of Chemical Engineering, The Ohio State University, USA, (2004).

8. A. Garud, D. Singh and N. Garud, Int. Curr. Pharm. J., 1, 384 (2012); https://doi.org/10.3329/icpj.v1i11.12065.

9. C. Vitorino, F.A. Carvalho, A.J. Almeida, J.J. Sousa and A.A.C.C. Pais, Colloids Surf. B Biointerfaces, 84, 117 (2011); https://doi.org/10.1016/j.colsurfb.2010.12.024.

10. L. Genç, G. Dikmen and G. Güney, J. Mater. Sci. Eng., 5, 468 (2011).

11. B. Lasa-Saracibar, A. Estella-Hermoso de Mendoza, M. Guada, C. DiosVieitez and M.J. Blanco-Prieto, Expert Opin. Drug Deliv., 9, 1245 (2012); https://doi.org/10.1517/17425247.2012.717928.

12. D. Hou, C. Xie, K. Huang and C. Zhu, Biomaterials, 24, 1781 (2003); https://doi.org/10.1016/S0142-9612(02)00578-1.

13. T.H. Le, J. Sci. Technol., 53, 159 (2015).

14. T.H.N. Le and N.Q.A. Phan, J. Sci. Technol., 53, 159 (2015). 